\title{
Newly Diagnosed Asthma in China: Initial Severity and Changes Over 1-year Management
}

\author{
Zhizhen Hu \\ Nanfang Hospital \\ Jianwei Xuan \\ Sun Yat-sen University \\ Haijin Zhao \\ Nanfang Hospital \\ Hangming Dong \\ Nanfang Hospital \\ Changhui $\mathrm{Yu}$ \\ Nanfang Hospital \\ Shaoxi Cai ( $\square$ hxkc@smu.edu.cn ) \\ Nanfang Hospital \\ Yue Gao \\ Centennial Scientific Co. Ltd \\ Liran Li \\ Centennial Scientific Co. Ltd \\ Xiaohan Hu \\ Centennial Scientific Co. Ltd
}

\section{Research Article}

Keywords: Asthma severity, new diagnosis, China, treatment

Posted Date: July 19th, 2021

DOl: https://doi.org/10.21203/rs.3.rs-659298/v1

License: (c) (i) This work is licensed under a Creative Commons Attribution 4.0 International License.

Read Full License 


\section{Abstract}

Background: The prevalence of adult asthma is increasing in China. However, there are no large sample, epidemiological data describing asthma severity at the time of new diagnosis and changes during followup management. Thus, the purpose of this study was to use a large health care database to examine asthma severity at initial diagnosis, and changes in severity over the first year of management.

Methods: Data of patients with a first diagnosis of asthma were extracted from the SuValue electronic medical database. Inclusion criteria were: 1) At least 14 years old at the time of first diagnosis; 2) Initial diagnosis from 2001 to March 2019;3) Followed-up for at least 12 months; 4) Had a follow-up visit every 3 months. Disease severity at diagnosis and at each follow-up visit, medications prescribed, and symptoms were collected and analysed.

Results: A total of 7,654 adult patients with newly diagnosed asthma from tertiary hospitals $(26.38 \%)$ and secondary hospitals (73.62\%) who were followed-up for at least 12 months were included. Approximately $54 \%$ were females, and the proportion of patients over 60 years old was the highest (38\%). Of all patients, $53.91 \%$ were not prescribed medications to control asthma, suggesting that these patients were mild asthma. Approximately $16 \%$ of patients were prescribed oral corticosteroid and/or inhaled corticosteroid and long-acting $\beta 2$-agonist combination, suggesting moderate to severe asthma. The proportions of patients with moderate and severe decreased during the first 6 months, and then the proportions remained stable. The proportion of patients with severe asthma remained stable from the 6th month onward. At the end of the year $2.7 \%$ of patients had severe asthma. Patients with mild asthma tended to continue to have mild asthma in the following 3 months ( $>76.19 \%$ ). However, of the patients with mild and moderate asthma at 3 months, $92.85 \%$ and $75.1 \%$, respectively, had a reduction in severity and had mild asthma at 12 months. On the other hand, $1.26 \%$ and $3.15 \%$, respectively, progressed to severe asthma by 12 months.

Conclusion: During the first year after an initial diagnosis of asthma patients diagnosed with mild asthma tended to not progress and remained stable with mild asthma over the year. The proportions of patients diagnosed with moderate and severe asthma remained stable over the year. Further study is needed to examine the clinical features of newly diagnosed patients with severe asthma who do not experience a reduction in severity in order to target these patients for more intensive treatment and reduce the disease burden.

\section{Background}

Bronchial asthma is a common chronic respiratory disease, and its prevalence is increasing worldwide. According to the 2015 Global Burden of Disease (GBD) Study, there are 358 million people with asthma worldwide, an increase of $12.6 \%$ since 1990 [1]. The Global Initiative for Asthma, Global Strategy for Asthma Management and Prevention (GINA) estimates that this number will increase to 400 million by 2025 [2]. 
In recent years, the rapid development of China's economy has accelerated urbanization resulting in major changes to people living environment and lifestyle. The prevalence of asthma among children $\leq 14$ years of age in China's urban areas was 1.54\% in 2000 [3], and 3.02\% in 2010 [4], an increase of $96.1 \%$ in 10 years. The reported incidence of adult asthma varies due to differences in sampling methods used by different epidemiological surveys and the definitions of asthma. The results of the China Asthma and Risk Factors Epidemiologic Investigation Study (CARE Study) conducted in 8 provinces and cities of 7 administrative regions in China from 2010 to 2011 showed that the prevalence of asthma diagnosed by doctors in people over 14 years old was $1.24 \%$, and $26 \%$ of the patients with asthma were newly diagnosed [5]. The China Pulmonary Health (CPH) Study was conducted from 2012 to 2015 in 10 provinces and cities of China and included 160 urban and rural sites, and reported the prevalence of asthma in persons $\geq 20$ years old was $4.2 \%$ [6].

Asthma severity is commonly assessed retrospectively based medications prescribed [2], and changes of prescriptions at follow-up visits can be used to indicate a worsening or improvement of asthma severity [7-9]. To date, however, there are no large sample epidemiological data from China describing asthma severity at the time of new diagnosis and changes under follow-up management. A prior a cross-sectional study showed that of 2,034 persons diagnosed with asthma, $6 \%$ were considered to have severe disease [10]. In another cross-sectional survey, $75 \%$ of 1,191 self-reported respondents were deemed to have mild asthma based on the medications they were prescribed [11].

Thus, the purpose of this study was to use a large health care database to examine asthma severity at initial diagnosis, and changes in severity over the first year of management. These results may help optimize asthma management and the allocation of medical resources.

\section{Methods}

\subsection{Data Source}

The SuValue health information database is a structured, electronic hospital medical record system constructed and based on ambulatory and hospitalization encounters that contains de-identified patient data of all diseases. The database captures the full continuum of treatment information for more than 90 million patients from 207 hospitals in 18 provinces in China. The patient data can be longitudinally tracked, and includes demographics information, diagnoses, laboratory testing results, medications prescribed, procedures performed, and the costs for inpatient, outpatient, and emergency department care. The database was constructed and is available for research purposes with the full authorization of each participating hospital. Centennial Scientific Co. Ltd., has dedicated access to the SuValue database.

\subsection{Study Design and population}

This was a retrospective study of newly diagnosed asthma patients. Eligible patients were at least 14 years old at the initial diagnosis of asthma (ICD-10-CM: J45) (index date) from 2001 to March 2019, and had at least 2 non-asthma related clinic visits separated by at least 30 days over the 1-year pre-index 
period to ensure the continuity of care [12]. Patients included in the study were followed-up for at least 12 months for the date of the initial diagnosis (Fig. 1).

This study was designed, implemented, and reported in accordance with the Strengthening the Reporting of Observational Studies in Epidemiology (STROBE) guidelines [13]. Only existing data from which all personally identifiable information was removed by the database provider prior to receipt by the study team was used in this study. Thus, informed patient consent and institutional review board/ethics committee approval were not required as indicated by the local medical research policy for using electronic medical records.

\subsection{Measurement}

Patient demographic data and medications prescribed at the index date were extracted from medical records. A drug-based severity classification method was used according to the GINA 2021 stepwise treatment recommendations [2]. Patients with asthma were categorized based on the intensity of asthma therapy prescribed into those with severe, moderate, and mild asthma. As defined by the Chinese Guidelines for Bronchial Asthma Prevention and Management 2020, patients with severe asthma were defined as those who were prescribed a medium/high-dose inhaled corticosteroid and long-acting $\beta 2$ agonist combination (ICS/LABA), or medium/high-dose ICS/LABA plus long-acting muscarinic antagonist (LAMA), or high-dose inhaled corticosteroid (ICS) plus leukotriene receptor antagonist or theophylline, and with additional anti-immunoglobulin E monoclonal antibody, LAMA, or oral corticosteroid. Patients with moderate asthma were defined as those who were prescribed a low-dose ICS/LABA, or medium/high-dose ICS, or low-does ICS plus leukotriene receptor antagonist (LTRA) or theophylline. Patients with mild asthma were defined as those not meeting the definition of either severe asthma or moderate asthma.

The proportions of patients with different asthma severities were recorded. Asthma severity classifications were summarized for each consecutive 3-month time window as an Observation Window Unit (OWU) from the index date to the end of 12-month follow-up. Patients were included in an OWU if they were seen within 1 month before or 1 month after the OWU. In situations in which multiple medications were recorded for a given $\mathrm{OWU}$, asthma severity classification was based on the last prescription (category and dosage) for the OWU.

\subsection{Statistics Analysis}

Data were reported as mean \pm standard deviation, or count (percentage). Subgroup analysis was carried out among the subsets of population with the index asthma visits from the hospitals of different tiers to compare their treatment patterns by using the Chi-square /Fisher exact test. The unequal variance twosample t-test was used for continuous data. All analyses were 2-tailed, and values of $P<0.05$ were considered to indicate statistical significance.

\section{Results}




\subsection{Study Population and Patient Characteristics}

A total of 7,654 patients meeting the inclusion criteria were identified in the database and included in the analysis. A flow diagram of patient selection and exclusion is shown in Figure 2. The mean age of the included patients was 52 years, and $54.18 \%$ were females. Of the patients, $37.66 \%$ were $>60$ years of age, accounting for the single largest proportion of patients, and $26.39 \%$ were 14 and 40 years old. Patients from tertiary hospitals accounted for $26.38 \%$ of the total, and those from secondary hospitals accounted for $73.62 \%$ (Table 1 ). Approximately $65 \%$ of patients were treated in internal medicine departments, $17 \%$ in emergency departments, and $11 \%$ in departments devoted to traditional Chinese medicine; however, the distributions of the departments where patients were treated varied with the level of care of the hospital. For example, in tertiary hospitals, patients were primarily treated in the respiratory medicine department (70.74\%) and internal medicine department $(29.26 \%)$. Whereas in secondary hospitals patients were treated in the internal medicine department (59.40\%), emergency department (21.22\%), and traditional Chinese medicine department (12.87\%) (Table 2).

\subsection{Initial Severity and symptom}

Of the 7,654 patients, $53.91 \%$ were not prescribed asthma control medications at the initial visit, suggesting that these patients had only mild intermittent symptoms $(<2$ times per month before diagnosis. On the other hand, $15.84 \%$ received medications including OCS and ICS-LABA, suggesting a more severe condition with symptoms prior to diagnosis (Table 3 ).

\subsection{Subsequent Severity}

Over the year of observation, the overall proportions of patients with moderate and severe decreased during the first 6 months, and then the proportions remained stable for the last 6 -month period. The proportion of patients with severe asthma remained stable from the 6th month onward. In the 4 observation windows $(3,6,9$, and 12 months), the mild asthma rates were $75.99 \%, 88.75 \%, 89.66 \%$, and $90.15 \%$, respectively, and the severe asthma rates were $5.33 \%, 2.56 \%, 2.55 \%$, and $2.7 \%$, respectively. At the end of the year, the proportion of patients with moderate and severe asthma was about $10 \%$, among which $2.7 \%$ had severe asthma (Figure 3).

During the 12-month follow-up, there were changes in asthma severity in some patients. Patients with mild asthma tended to continue to have mild asthma in the following 3 months ( $>76.19 \%)$. However, of the patients with mild and moderate asthma at 3 months, $92.85 \%$ and $75.1 \%$, respectively, had a reduction in severity and had mild asthma at 12 months. On the other hand, $1.26 \%$ and $3.15 \%$, respectively, progressed to severe asthma by 12 months (Figure 4).

\section{Discussion}

To the best of our knowledge, this is the first study performed in China investigating initial asthma severity at diagnosis, and changes in severity over the first year of management. Our results showed that 
after 1 year nearly $10 \%$ of patients had moderate or severe asthma. Our study represents actual clinical practice in China of a heterogeneous adult and adolescent populations treated in both tertiary and secondary hospitals with a wide regional coverage. The results of our study spotlight real-world management of asthma, and complement previous data from cross-sectional population-based surveys.

Unlike other chronic diseases, there are no objective markers of underlying asthma disease severity; however, asthma severity can be assessed when a patient has been on controller treatment for several months. In this study that included 7,654 newly diagnosed asthma patients, the prevalence of severe asthma was stable during a 1-year follow-up period. Our result of a frequency of $2.7 \%$ of patients having severe asthma at 1 year is similar to that of $2.4 \%$ reported in a retrospective study performed in Japan that used a nationwide health care claims database [14], but somewhat lower than the commonly reported prevalence of $5-10 \%[15,16]$. In a prior cross-sectional study in China [10], the investigators used different criteria for determining asthma severity that what was used in our study [17]. Data used in our study were obtained from continuous outpatient records, and there were no lapses of treatment or missing data with respect to any of our included patients.

Population-based studies and longitudinal studies in other countries help in understanding changes in asthma over time and associations with initial disease severity. A cross-sectional survey of 3,509 children aged 5-14 years living in rural and urban regions of Canada found a difference in childhood asthma prevalence between urban and rural locations, and once a child has asthma certain rural exposures may aggravate the disease [18]. A population-based survey of over 12,000 adolescents in France showed that the prevalence of 'ever asthma' was higher among boys, whereas severe asthma was associated with early onset and female sex [19]. Important for the allocation of health care resources, the authors found that asthmatic adolescents required more health care in terms of medication use, consultations and hospitalizations other than for asthma than other adolescents, and this relation was strongest among severe asthmatics. A Danish study followed asthmatics for over 30 years, and reported that female sex, previous severe exacerbation(s), and older age at baseline were associated with uncontrolled asthma at follow-up, and a blood-eosinophil count $\geq 0.3 \times 10^{9} \mathrm{~L}$ and being prescribed an ICS at baseline were associated with being prescribed a medium- or high-dose ICS at follow-up [20]. The authors concluded that asthma rarely remits in adults, especially in individuals with longer duration and more severe disease, and worse initial disease is associated with uncontrolled asthma at follow-up.

GINA guidelines provide specific recommendations for choices of pharmacotherapy and frequency of regular follow-up for monitoring asthma control and adjusting therapy as needed. However, in many cases the goals of an individual patient will differ from medical goals. Poor adherence to therapy is common in patients with asthma, and is often associated with increased use of health care resources, morbidity, and mortality, and this includes patients in China with asthma [21,22]. A clear explanation of asthma and disease severity by a physician can improve a patient's trust and confidence in treatment. Asthma severity is not a static feature and may change overtime, and in our study approximately $90 \%$ of patients had mild asthma at 12 months, even though a marked proportion of patients had moderate or severe asthma during the first 6 months. Importantly our results showed that in general patients 
diagnosed initially with mild asthma tended to continue to have mild asthma and not progress in disease severity.

Our results showed that a small group of patients developed severe asthma over the year, and some patients with severe asthma did not have a reduction in severity over the year. As such, further study is needed to examine the clinical features of newly diagnosed patients with severe asthma who do not experience a reduction in severity in order to target these patients for more intensive treatment and reduce the disease burden.

There are both strengths and weaknesses of using a health information database. One of the main advantages is that it provides health care data from a large number of people. Another advantage is that all outpatient visits and hospitalizations can be identified. This allows follow-up of individual patients; most epidemical studies are cross-sectional without follow-up data. These advantages enabled the research to determine the reality of asthma management, and complement the limitations of previous studies performed in a hospital-based setting. Furthermore, our study only included individuals who were actively engaged with their health care providers, i.e., we only included individuals who had been in regular contact with the health care system for ailments other than asthma before the index date. By doing so, we minimized the frequency of patients that may have been lost to follow-up for various reasons, such as loss of health insurance coverage.

On the other hand, there are limitations inherent is studies that are based on data from medical records. Data retrieval is limited to the variables registered in the database, and medication use is based on medications prescribed and this does not necessarily reflect how patients actually use their medications. Our study classified asthma severity according to medications prescribed, not clinical data, and did not assess medication adherence. These shortcomings may have resulted in the misclassification of asthma severity in some patients. In addition, using asthma medications as a proxy for asthma severity relies on physician adherence to current practice guidelines [23]. Deviation of asthma treatment from guidelines may result in inaccurate estimations of asthma severity and other potential biases [24].

\section{Conclusions}

Our results showed that during the first year after an initial diagnosis of asthma patients diagnosed with mild asthma tended to not progress and remained stable with mild asthma over the year. The proportions of patients diagnosed with moderate and severe asthma remained stable over the year. As such, further study is needed to examine the clinical features of newly diagnosed patients with severe asthma who do not experience a reduction in severity in order to target these patients for more intensive treatment and reduce the disease burden.

\section{Abbreviations}

AR: allergic rhinitis 
GINA: The Global Initiative for Asthma

ICS: inhaled corticosteroid

ICS-LABA: Inhaled corticosteroid and long-acting $\beta 2$-agonist combination

LAMA: long-acting muscarinic antagonist

LTRA: leukotriene receptor antagonist

MCS: mast cell stabilizer

OCS: oral corticosteroid

OWU: Observation Window Unit

SABA: short-acting $\beta 2$-agonist

SAMA: short-acting muscarinic antagonist

TCM: Traditional Chinese medicine

\section{Declarations}

\section{Ethics approval and consent to participate: Not applicable}

This is a retrospective analysis from a structured electronic hospital medical record system constructed and based on ambulatory and hospitalization encounters with de-identified patient level data, and not involving human participants, human data or human tissue

\section{Consent for publication: Not applicable}

This manuscript does Not contain any individual person's data in any form (including individual details, images or videos), consent to publish.

\section{Availability of data and material}

Raw data used in this analysis is available from the corresponding author upon request.

\section{Funding}

This study was supported by the Scientific and Technological Project of Guangdong Province ( 2017B020226006) and National Natural Science Foundation of China $(81970032,81770033 \llbracket 81670026)$ . The funders of the study had a role in study design, data analysis, data interpretation, and writing of the report. The corresponding author had the final responsibility to submit this work for publication. Study 
conduct and data analysis were performed by Centennial Scientific Co. Ltd. No funding was provided to the employees of Centennial Scientific Co. Ltd for manuscript development.

\section{Competing interests}

The authors declare that they have no competing interests.

\section{Authors' contributions}

$\mathrm{ZH}, \mathrm{SC}, \mathrm{JX}$ and $\mathrm{YG}$ were involved in the conception and design of the study, the acquisition of data, and the data analysis and interpretation. $\mathrm{XH}, \mathrm{HZ}, \mathrm{HD}, \mathrm{CY}$ and $\mathrm{XL}$ were involved in the data analysis and interpretation. All authors contributed to drafting and revising the article, gave final approval of the version to be published, and agree to be accountable for all aspects of the work.

\section{Acknowledgements: Not applicable}

\section{References}

1. GBD 2015 Chronic Respiratory Disease Collaborators. Global, regional, and national deaths, prevalence, disability-adjusted life years, and years lived with disability for chronic obstructive pulmonary disease and asthma, 1990-2015: a systematic analysis for the Global Burden of Disease Study 2015. Lancet Respir Med. 2017;5(9):691-706. doi: 10.1016/S2213-2600(17)30293-X

2. Global Initiative for Asthma, Global strategy for asthma management and prevention, Available from: www.ginasthma.org

3. National Cooperation Group on Childhood Asthma. A nationwide survey in China on the prevalence of asthma in urban children. Chin J Pediatr. 2003;41:123-127.

4. National Cooperation Group on Childhood Asthma. Third nationwide survey of childhood asthma in urban areas of China. Chin J Pediatr. 2013;51(10):729-735.

5. Lin J, Wang W, Chen P, Zhou X, Wan H, Yin K, Ma L, et al. Prevalence and risk factors of asthma in mainland China: The CARE study. Respir Med. 2018;137:48-54. doi: 10.1016/j.rmed.2018.02.010

6. Huang K, Yang T, Xu J, et al.; China Pulmonary Health (CPH) Study Group. Prevalence, risk factors, and management of asthma in China: a national cross-sectional study. Lancet. 2019;394(10196):407-418. doi: 10.1016/S0140-6736(19)31147-X

7. Reddel HK, Taylor DR, Bateman ED, Boulet LP, Boushey HA, Busse WW, Casale TB, et al. An official American Thoracic Society/European Respiratory Society statement: asthma control and exacerbations: standardizing endpoints for clinical asthma trials and clinical practice. Am J Respir Crit Care Med. 2009;180:59-99.

8. Taylor DR, Bateman ED, Boulet LP, Boushey HA, Busse WW, Casale TB, Chanez P, et al. A new perspective on concepts of asthma severity and control. Eur Respir J. 2008;32:545-54. 
9. Chung KF, Wenzel SE, Brozek JL, Bush A, Castro M, Sterk PJ, Adcock IM, et al. International ERS/ATS Guidelines on Definition, Evaluation and Treatment of Severe Asthma. Eur Respir J. 2014;43:343-373.

10. Su N, Lin JT, Wang WY, Chen P, Zhou X, Wan HY, Yin KS, et al. [A cross-section study of severe asthma in eight provinces of China]. Zhonghua Nei Ke Za Zhi. 2016;55(12):917-921. doi: 10.3760/cma.j.issn.0578-1426.2016.12.002 [Article in Chinese]

11. Ding B, DiBonaventura M, Karlsson N, Ling X. A cross-sectional assessment of the prevalence and burden of mild asthma in urban China using the 2010, 2012, and 2013 China National Health and Wellness Surveys. J Asthma. 2017;54(6):632-643.

12. Hernán MA, Robins JM. Using big data to emulate a target trial when a randomized trial is not available. Am J Epidemiol. 2016;183(8):758-764. doi: 10.1093/aje/kwv254

13. von Elm E, Altman DG, Egger M, Pocock SJ, Gøtzsche PC, Vandenbroucke JP; STROBE Initiative. The Strengthening the Reporting of Observational Studies in Epidemiology (STROBE) statement: guidelines for reporting observational studies. J Clin Epidemiol. 2008;61(4):344-349.

14. Sato K, Ohno T, Ishii T, Ito C, Kaise T. The prevalence, characteristics, and patient burden of severe asthma determined by using a Japan health care claims database. Clin Ther. 2019;41(11):22392251. doi: 10.1016/j.clinthera.2019.08.015

15. Bel EH, Sousa A, Fleming L, Bush A, Chung KF, Versnel J, et al. Diagnosis and definition of severe refractory asthma: an international consensus statement from the innovative medicine initiative (IMI). Thorax. 2011;66(10):910-917.

16. O'Byrne PM, Naji N, Gauvreau GM. Severe asthma: future treatments. Clin Exp Allergy. 2012;42(5):706-711.

17. Asthma Workgroup, Chinese Thoracic Society Expert consensus on diagnosis and management of refractory asthma. Chin J Tubere Respir Dis. 2010;33(8):572-577.

18. Lawson JA, Rennie DC, Cockcroft DW, Dyck R, Afanasieva A, Oluwole O, Afsana J. Childhood asthma, asthma severity indicators, and related conditions along an urban-rural gradient: a cross-sectional study. BMC Pulm Med. 2017;17(1):4. doi: 10.1186/s12890-016-0355-5

19. Moreau D, Ledoux S, Choquet M, Annesi-Maesano I. Prevalence and severity of asthma in adolescents in France. Cross-sectional and retrospective analyses of a large population-based sample. Int J Tuberc Lung Dis. 2000;4(7):639-648.

20. Tupper OD, Håkansson KEJ, Ulrik CS. Remission and changes in severity over 30 years in an adult asthma cohort. J Allergy Clin Immunol Pract. 2021;9(4):1595-1603.e5. doi:

10.1016/j.jaip.2020.11.013.

21. Boulet LP, Vervloet D, Magar Y, Foster JM. Adherence: the goal to control asthma. Clin Chest Med. 2012;33(3):405-417.

22. Yuan Y, Li B, Huang M, Peng X, Zhao W, Ye Y, Zhang P, et al. Fractional exhaled nitric oxide was not associated with the future risk of exacerbations in Chinese asthmatics: a non-interventional 1-year real-world study. J Thorac Dis. 2019;11(6):2438-2447. 
23. Pont LG, van der Werf GT, Denig P, Haaijer-Ruskamp FM. Identifying general practice patients diagnosed with asthma and their exacerbation episodes from prescribing data. Eur J Clin Pharmacol. 2002;57(11):819-825.

24. Hasford J, Uricher J, Tauscher M, Bramlage P, Virchow JC. Persistence with asthma treatment is low in Germany especially for controller medication - a population based study of 483,051 patients. Allergy. 2010;65(3):347-354. doi: 10.1111/j.1398-9995.2009.02161.x

\section{Tables}

Table 1. Patient characteristics $(\mathrm{N}=7,654)$

\begin{tabular}{|ll|}
\hline Sex & \\
\hline Male & $3,507(45.82)$ \\
\hline Female & $4,147(54.18)$ \\
\hline Age (years) & $52 \pm 17.08$ \\
\hline Age proportion (years) & \\
\hline $14-40$ & $2,020(26.39)$ \\
$>40-60$ & $2,752(35.96)$ \\
$>60$ & $2,882(37.66)$ \\
\hline Hospital & $2,019(26.38)$ \\
\hline Tertiary & $5,635(73.62)$ \\
\hline
\end{tabular}

Age reported as mean \pm standard deviation; other data as count (percentage).

Table 2. Distributions of locations of initial asthma diagnosis 


\begin{tabular}{|llll|}
\hline & All Patients & Tertiary Hospital & Secondary Hospital \\
\hline & $\mathbf{7 , 6 5 4}(\mathbf{1 0 0} \%)$ & $\mathbf{2 0 1 9}(\mathbf{2 6 . 3 8 \% )}$ & $\mathbf{5 6 3 5}(\mathbf{7 3 . 6 2 \% )}$ \\
\hline Department & & & \\
\hline Internal medicine & $4,967(64.89 \%)$ & $1,620(80.24 \%)$ & $3,347(59.40 \%)$ \\
\hline Respiratory & $2,033(26.56 \%)$ & $1,146(56.76 \%)$ & $887(15.74 \%)$ \\
\hline Non-respiratory & $2,934(38.33 \%)$ & $474(23.48 \%)$ & $2,460(43.66 \%)$ \\
\hline Emergency department & $1,331(17.39 \%)$ & $135(6.69 \%)$ & $1,196(21.22 \%)$ \\
\hline Chinese medicine & $863(11.28 \%)$ & $138(6.84 \%)$ & $725(12.87 \%)$ \\
\hline Otolaryngology & $270(3.53 \%)$ & $29(1.44 \%)$ & $241(4.28 \%)$ \\
\hline Surgical & $112(1.46 \%)$ & $53(2.63 \%)$ & $59(1.05 \%)$ \\
\hline Pediatric & $96(1.25 \%)$ & $41(2.03 \%)$ & $55(0.98 \%)$ \\
\hline Obstetrics and Gynecology & $15(0.20 \%)$ & $3(0.15 \%)$ & $12(0.21 \%)$ \\
\hline
\end{tabular}

Table 3. Treatments at the initial asthma diagnosis 


\begin{tabular}{|c|c|c|c|c|}
\hline & $\begin{array}{l}\text { All Hospitals } \\
\begin{array}{l}N=7,654 \\
(100 \%)\end{array}\end{array}$ & $\begin{array}{l}\text { Tertiary } \\
\text { Hospitals } \\
n=2,019 \\
(26.38 \%)\end{array}$ & $\begin{array}{l}\text { Secondary } \\
\text { Hospitals } \\
\\
n=5,635 \\
(73.62 \%)\end{array}$ & $\begin{array}{l}\text { P value } \\
\text { (Tertiary } \\
\text { vs. } \\
\text { Secondary) }\end{array}$ \\
\hline Controller & $3,527(46.09 \%)$ & $1,226(60.73 \%)$ & $2,301(40.84 \%)$ & \\
\hline OCS containing & $264(3.45 \%)$ & $64(3.18 \%)$ & $200(3.55 \%)$ & $<0.001$ \\
\hline ICS-LABA containing & $948(12.39 \%)$ & $482(23.87 \%)$ & $466(8.27 \%)$ & \\
\hline ICS containing & $152(1.99 \%)$ & $45(2.24 \%)$ & $107(1.90 \%)$ & \\
\hline LTRA containing & $1,009(13.19 \%)$ & $398(19.72 \%)$ & $611(10.85 \%)$ & \\
\hline Theophylline containing & $537(7.01 \%)$ & $125(6.19 \%)$ & $412(7.31 \%)$ & \\
\hline Others (MCS) & $617(8.06 \%)$ & $112(5.53 \%)$ & $505(8.96 \%)$ & \\
\hline Anti-asthmatic medications & $1,780(23.25 \%)$ & $255(12.61 \%)$ & $1525(27.07 \%)$ & \\
\hline SABA containing & $587(7.66 \%)$ & $99(4.93 \%)$ & $487(8.64 \%)$ & \\
\hline $\begin{array}{l}\text { Other anti-asthmatic } \\
\text { medications }\end{array}$ & $1,193(15.59 \%)$ & $155(7.68 \%)$ & $1,038(18.42 \%)$ & \\
\hline Symptomatic medications & $1,105(14.43 \%)$ & $262(12.97 \%)$ & $843(14.96 \%)$ & \\
\hline No medication prescribed & $1,242(16.23 \%)$ & $276(13.69 \%)$ & $966(17.14 \%)$ & \\
\hline
\end{tabular}

OCS: oral corticosteroid; ICS-LABA: inhaled corticosteroid and long-acting $\beta 2$-agonist combination; ICS: inhaled corticosteroid; LTRA: leukotriene receptor antagonist; MCS: mast cell stabilizers; SABA: shortacting $\beta 2$-agonist; TCM: Traditional Chinese medicine.

Patients were categorized by the highest preferred treatment of each step; e.g., categorized as OCS if the combination of OCS and ICS-LABA was prescribed.

Anti-asthmatic medications included SAMA, SABA/SAMA combination, short-acting xanthine derivatives, short course ( $\leq 7$ days), and injected/oral/nebulized corticosteroids.

Symptomatic medications included TCM, antitussive, mucolytics, cough-mucolytic combination, antihistamine, and antibots.

\section{Figures}




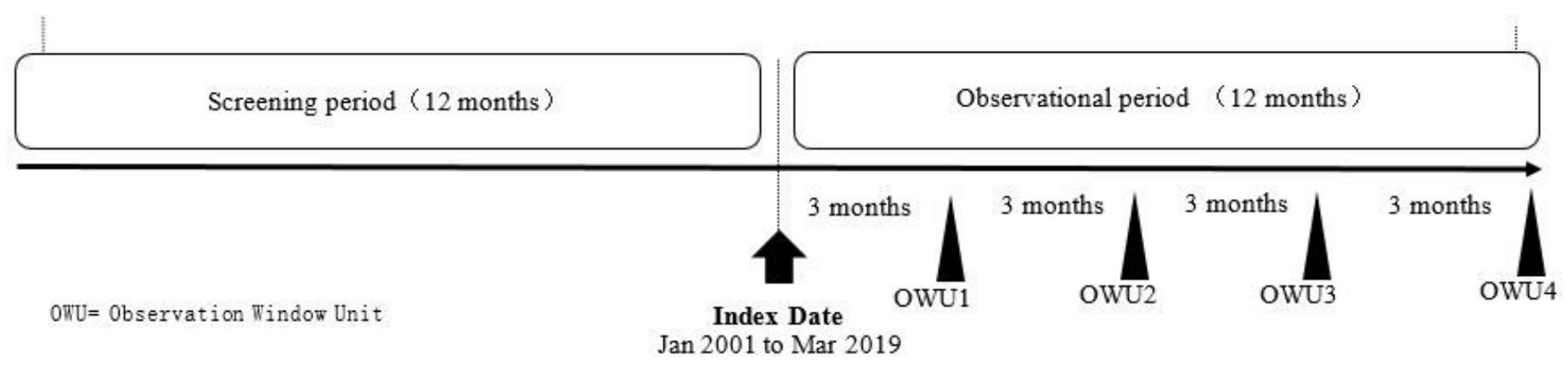

\section{Figure 1}

\section{Study design}

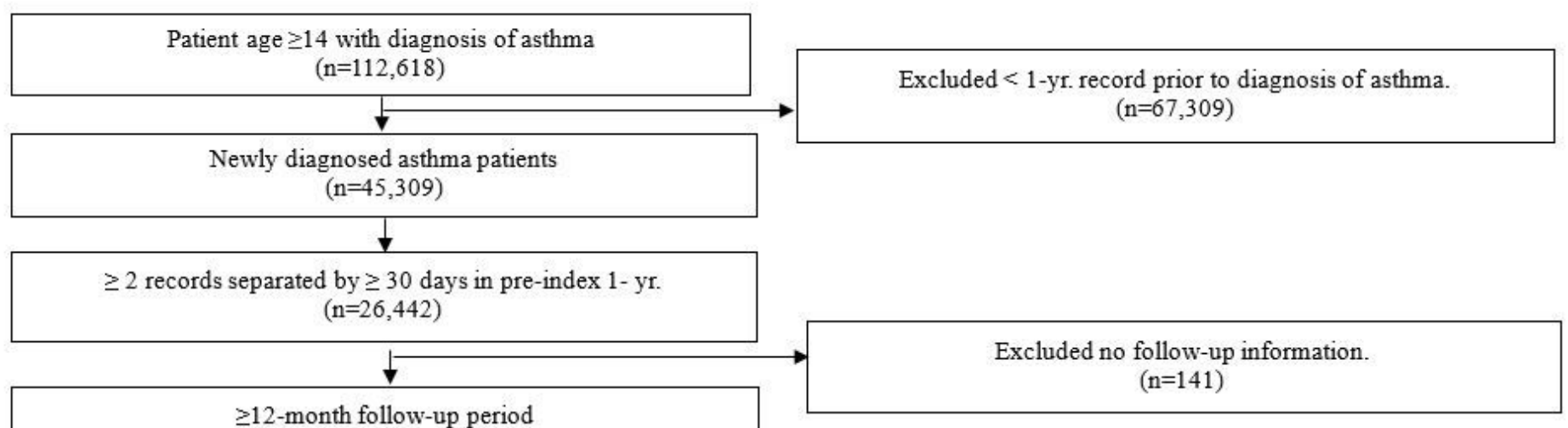

\section{Figure 2}

\section{Study design}

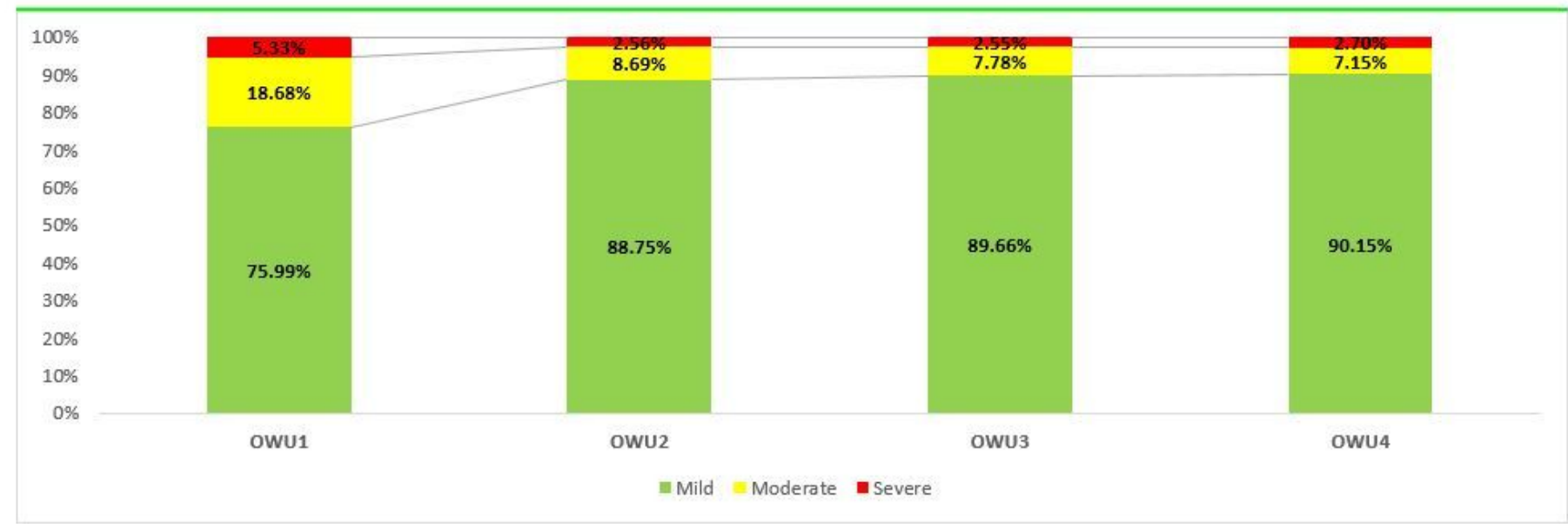

\section{Note:}

1. OWU: Observation Window Unit, 1 OWU $=3$ months

2. Asthma severity classification was based on the last prescription of controller medication

3. 1-month grace period before and after the OWU was permitted to account for patients' early or late visit. 
Figure 3

Distribution of Severity in Each 3-month OWU

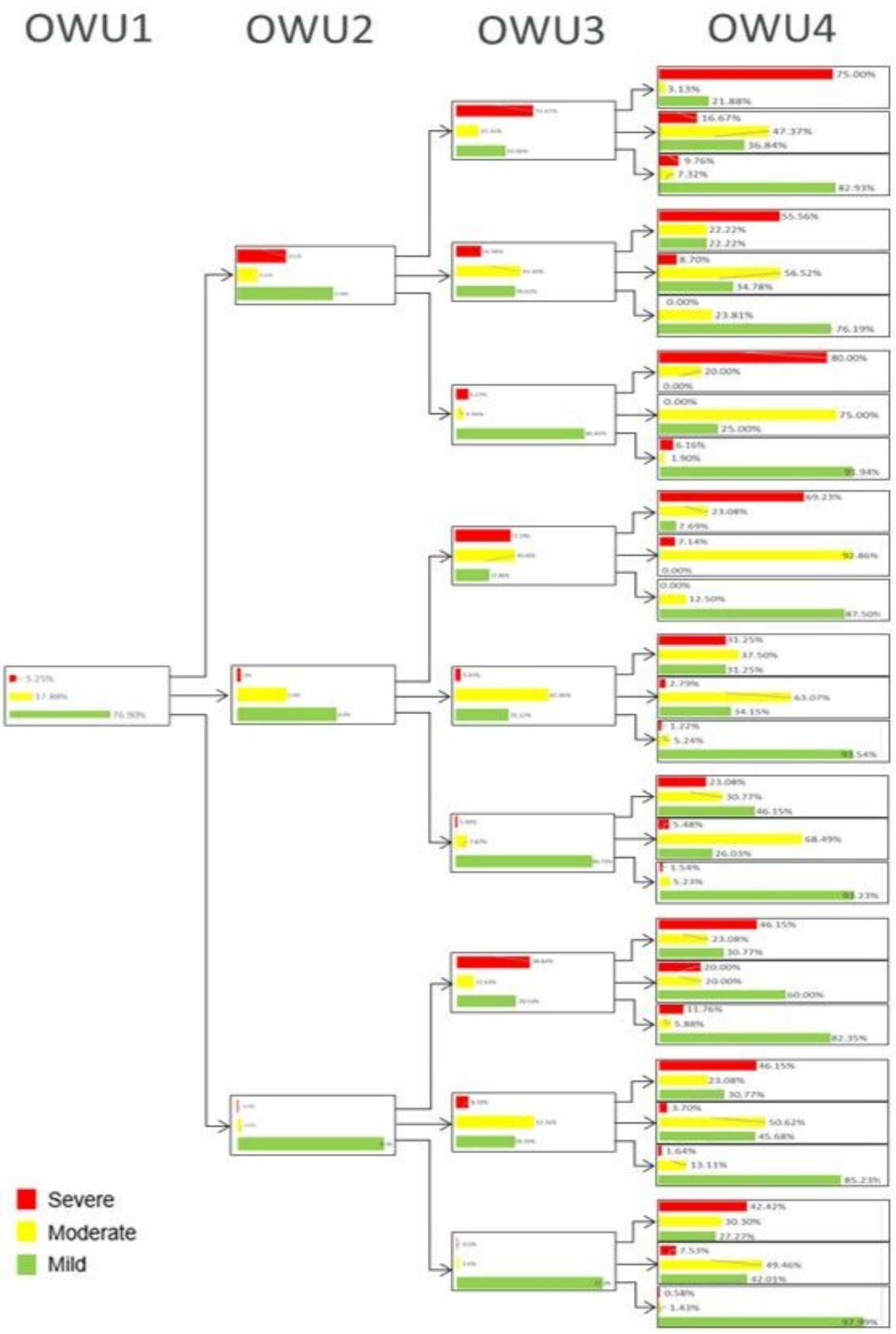

Figure 4

Severity change in Each 3-month OWU 